\title{
Formación de capital humano con vistas al desarrollo de ciudades impactadas por el Corredor Vial Bioceánico
}

\author{
Formation of human capital with a view to the development of cities impacted \\ by the Bioceanic Road Corridor
}

\author{
Formação de capital humano com vistas ao desenvolvimento das cidades \\ impactadas pelo Corredor Rodoviário Bioceânico
}

\author{
Edilene Maria de Oliveira ${ }^{1}$ \\ Arlinda Cantero Dorsa ${ }^{2}$ \\ Bruno de Matos Farias ${ }^{3}$ \\ Maria Geralda de Miranda ${ }^{3}$
}

Recibido el 5 de jul. de 2021; revisado e aprobado el 7 de sept. de 2021; aceptado el 28 de sept. de 2021 DOI: http://dx.doi.org/10.20435/inter.v22i4.3443

\begin{abstract}
Resumen: El Corredor Vial Bioceánico unirá los océanos Pacífico y Atlántico, pasando por Brasil, Paraguay, Argentina hasta llegar a los puertos de Chile. Esta iniciativa traerá importantes cambios económicos, sociales y ambientales al Estado de Mato Grosso do Sul. Si este corredor es una ruta para la integración de América Latina y una política estratégica, los países involucrados son enfáticos en reconocer que, en el suelo, será un corredor para el paso de mercancías, sino una que traerá desarrollo a todas las regiones que se encuentran rastro de esta ruta. Las ciudades del Estado de Mato Grosso do Sul donde habrá acceso al corredor, a excepción de la Capital Campo Grande, son pequeñas ciudades que pueden impactarse directamente en diversos sectores, como infraestructura, turismo, ocio. y hostelería y gestión empresarial. Habrá necesidades para atender los requerimientos laborales que se vayan planteando, requiriendo formación y cualificación profesional para atender las nuevas demandas laborales. Así, este estudio tiene como objetivo aportar una visión del desarrollo en sus diversas concepciones, así como señalar posibilidades de cualificación profesional en numerosas áreas que deberán ser priorizadas en el corto, medio y largo plazo, para que el Estado de Mato Grosso do Sul logre desarrollar su mano de obra local. A partir de una investigación bibliográfica y documental, se concluyó que la oferta de cursos técnicos y de educación inicial y continuada para jóvenes y personas en general, puede servir positivamente a los municipios de Mato Grosso do Sul por donde pasa la Ruta de la Carretera Bioceánica, formando y capacitando su capital humano, con vistas al desarrollo de las localidades.
\end{abstract} Palabras clave: Corredor de Bioceánico; desarrollo; capital humano; formación profesional.

Abstract: The Bioceanic Road Corridor will link the Pacific and Atlantic oceans, passing through Brazil, Paraguay, Argentina until reaching the ports of Chile. This undertaking will bring significant economic, social and environmental changes to the State of Mato Grosso do Sul. As much as this corridor is a route for the integration of Latin America and a strategic policy, the countries involved are emphatic in recognizing that it will not only be a corridor for the passage of goods, but will bring development to all regions that are in the trace of this route. The cities in the State of Mato Grosso do Sul, where the corridor will have access, with the exception of the Capital Campo Grande, are small cities that may be directly impacted in various sectors, such as infrastructure, such as infrastructure, tourism, leisure and hospitality and business management. There will be needs to attend to labor issues that will arise, requiring professional training and qualifications to meet the new work demands. Thus, this study aims to bring scores on development in its various conceptions, as well as pointing out possibilities of professional qualification in numerous areas that will have to be prioritized in the short, medium and long term, so that the State of Mato Grosso do Sul can develop its local labor. Based on a bibliographical and documentary research, it was concluded that the offer of technical courses and initial and continuing education for young people and people in general, may positively serve the municipalities of Mato Grosso do Sul through which the Bioceanic Highway Route passes, forming and qualifying its human capital, with a view to the development of localities.

Keywords: Bioceanic Corridor; development; human capital; professional qualification.

\footnotetext{
${ }^{1}$ Instituto Federal de Mato Grosso do Sul, Campo Grande, Mato Grosso do Sul, Brasil.

${ }^{2}$ Universidade Católica Dom Bosco, Campo Grande, Mato Grosso do Sul, Brasil .

${ }^{3}$ Centro Universitário Augusto Motta, Rio de Janeiro, Rio de Janeiro, Brasil.
} 


\begin{abstract}
Resumo: O Corredor Rodoviário Bioceânico ligará os oceanos Pacífico ao Atlântico, passando pelo Brasil, Paraguai, Argentina até chegar aos portos do Chile. Tal empreendimento trará para o Estado de Mato Grosso do Sul significativas mudanças econômicas, sociais e ambientais. Por mais que este corredor seja uma rota de integração da América-latina e uma política estratégica, os países envolvidos são enfáticos em reconhecer que não somente será um corredor de passagem de mercadorias, mas sim trará o desenvolvimento a todas as regiões que se encontram no traçado desta rota. As cidades do Estado de Mato Grosso do Sul, onde o corredor terá acesso, com exceção da Capital Campo Grande, são cidades de pequeno porte e que poderão ser impactadas diretamente em diversos setores, como o de infraestrutura, turismo, lazer e hotelaria e gestão de negócios. Haverá necessidade de atendimento a questões laborais que surgirão, requerendo formações e qualificações profissionais para atendimento às novas demandas de trabalho. Assim, este estudo objetiva trazer pontuações sobre o desenvolvimento em suas diversas concepções, como também apontar possibilidades de qualificação profissional em inúmeras áreas que terão que ser priorizadas a curto, médio e longo prazo, para que o Estado de Mato Grosso do Sul consiga desenvolver sua mão de obra local. A partir de pesquisa bibliográfica e documental, concluiu-se que as ofertas de cursos técnicos e formação inicial e continuada para jovens e pessoas em gerais, poderão atender de forma positiva aos municípios de Mato Grosso do Sul por onde passar a Rota Rodoviária Bioceânica, formando e qualificando seu capital humano, com vistas ao desenvolvimento das localidades.
\end{abstract}

Palavras-chave: Corredor Bioceânico; desenvolvimento; capital humano; formação profissional.

\title{
1 CONSIDERACIONES INICIALES
}

A partir de una reunión de presidentes sudamericanos en el año 2000, se planteó la posibilidad de abrir un Corredor Bioceánico que permitiera la integración del continente sudamericano con el Océano Pacífico para la destinación de la producción de los países involucrados, procurando así, a la minimización de los costos financieros y operativos debido a la reducción de la distancia entre estos países y Asia, destino de gran parte de los productos exportados.

Dicha denominación fue utilizada inicialmente por las autoridades públicas, como corredores de transporte o destinación de granos, y anteriormente de Ejes de Integración y desarrollo (denominación antigua en razón de premisas designadas por autoridades gubernamentales).

La formalización de este Corredor Vial Bioceánico, sin embargo, solo se llevó a cabo en diciembre de 2015, a través de la Declaración de Asunción, documento firmado por los presidentes de Brasil, Chile, Argentina y Paraguay, creando así un Grupo de Trabajo (GT), el cual tuvo como objetivo las implementaciones de acciones dirigidas a la infraestructura de las regiones por donde pasará el corredor (CASTRO, 2019).

Se entiende, por tanto, que un corredor que estratégicamente conectará los océanos Atlántico, a partir del puerto de Santos, y el océano Pacífico, llegando a los puertos chilenos de Antofagasta e Iquique, con destinos a los puertos asiáticos, a la Costa Este americana, a Canadá y al Oriente Medio.

La ruta Bioceánica (Corredor Bioceánico) se basa en la integración latinoamericana, así como si se trata de una política estratégica para todas las regiones implicadas, no obstante, no es percibida por los gobiernos como siendo solamente una ruta de paso de mercancías, sino una ruta de oportunidades que posibilitará a las localidades el desarrollo en sus conceptos diversos.

Al pasar por el estado de Mato Grosso do Sul, con la excepción del capital Campo Grande, provocará los impactos directos en la infraestructura física de las ciudades de pequeño porte y exigirá cambios expresivos con respecto a la formación y a la calificación para la atención a la nueva realidad vivida, foco principal de este artículo.

El presente artículo es una investigación exploratoria, bibliográfica y documental con enfoque cuantitativo, con vistas a presentar posibilidades de formación a través de cursos rápidos, 
así como formación técnica, que puede ser tanto posterior como concomitante, que podrán asistir a los municipios en el desarrollo y/o perfeccionamiento de conocimiento y habilidades de sus trabajadores.

La preparación del ciudadano, a través de la formación laboral, se reflejará en la mejora o adquisición de nuevas habilidades, cuya mano de obra capacitada estará disponible para realizar las diversas funciones derivadas de las nuevas demandas de las localidades impactadas por el Corredor Bioceánico, solo así estas localidades serán capaces de alcanzar el desarrollo en sus diversas perspectivas.

\section{EL CORREDOR VIAL BIOCEÁNICO EN MATO GROSSO DO SUL - CIUDADES IMPACTADAS}

En el estado de Mato Grosso do Sul, como punto de partida está la capital del estado, Campo Grande, rumbo a Porto Murtinho, ciudad fronteriza con Carmelo Peralta en Paraguay, donde los límites se dan por medio pluvial. Así, Brasil y Paraguay construirán un puente sobre el Río Paraguay, con presupuestos ya aprobados. Para el Gobierno del Estado de Mato Grosso do Sul (2021a) esta obra representa el eslabón principal de todo el Corredor Bioceánico.

Según informes de la prensa local y nacional, el flujo estimado de carga en ruta a países vecinos puede llegar hasta a 700 camiones al día (VIEGAS, 2019). Serán cargas de exportación para los países vecinos, para América del Norte, y para el otro lado del Océano Pacífico. Según el secretario de SEMAGROMS, "[...] la proyección para los años próximos es la de invertir en la diversificación de la base económica, facilitar la logística y mejorar la infraestructura" (BENITES, 2020, p. 1).

La expresión Ruta Bioceánica y Corredor Bioceánico en este contexto se ha utilizado por varios investigadores, gestores públicos, y en esa dualidad, podemos entonces considerar el Corredor Bioceánico, como un espacio físico de paso y la Ruta como un eslabón de integración humana, comercial, cultural y ambiental, con amplia relevancia para la academia, para el poder público y para la iniciativa privada.

En la realización del paso de Campo Grande a Porto Murtinho, la ruta pasará por diversas ciudades, las cuales serán impactadas directamente. Según Constantino (2019), si comtenplamos los dos caminos posibles entre Campo Grande y Porto Murtinho, serán 14 ciudades: Anastácio, Aquidauana, Bodoquena, Bonito, Campo Grande, Dois Irmãos do Buriti, Guia Lopes da Laguna, Jardim, Maracaju, Miranda, Nioaque, Porto Murtinho, Sidrolândia y Terenos.

De las ciudades mencionadas, con la excepción dela capital Campo Grande, todas las demás son ciudades de pequeño porte, que tendrán que ajustarse a los cambios que están por venir, una vez que durante todo el trayecto de la ruta Bioceánica, habrá necesidad de mejoría en la infraestructura, bien para la atención a conductores de camiones, que traerán las cargas para la exportación y los viajeros que utilizarán esta ruta, o incluso para el flujo turístico con las proyecciones de aumentos significativos. Además del transporte vial también es imperativa la mirada sobre los demás modales ferrocarril, por ríos, vial y aéreo, una vez que complementan la logística de la cadena de la exportación.

En este primer momento, es perceptible que, para atender a la cuestión vial, será nuclear que haya nuevas inversiones o incluso la ampliación de diversos servicios, tales como gasolineras y afines, servicios mecánicos, mejora y aumento de la red de hostelería, así como de restaurantes y demás servicios del sector de alimentación. La mejora y la extensión de la atención al turista, a 
los servicios de logística y de aduana, entre muchos otros sectores, tendrán que ajustarse, incluso en relación a los demás modales de ferrocarril, vías navegables y aéreo, bien como a las nuevas inversiones que surgirán, ya que la ruta Bioceánica se presenta como una gran oportunidad para emprender, puesto que, combinada con la expansión de estas actividades, será indispensable la disponibilidad de material humano calificado.

La ruta tiene un papel básico en la integración latinoamericana, pero también aparece como una política estratégica para estas regiones de cada uno de estos países. Hablamos de integración y desarrollo local de estas áreas. Es una ruta de oportunidades y de posibilidades para que los gobiernos nacionales y también la población, si se apropian de estos procesos de desarrollo económico y social. (BARROS, 2020, p. 8).

El Corredor Bioceánico es discutido por las autoridades como algo que no debe ser percibido solamente como un corredor de paso de mercancías, sino un corredor que traerá a todas las localidades implicadas la perspectiva del desarrollo económico, social, humano, así como conduciendo al desarrollo local.

\subsection{Conceptos sobre el desarrollo}

El término desarrollo para Baquero y Cremonese (2008) empezó a ser usado aún en el siglo XIX y se hacía presente en el ambiente social e intelectual de la modernidad. El término nació a partir de las revoluciones Francesa e Industrial y por medio de la constitución de los Estados Nacionales, centrada intelectualmente en los principios liberales, apuntando para la posibilidad de un inmenso progreso humano y material. Los autores también refieren que el término ocupó lugar de destaque al final de la Segunda Guerra Mundial, una vez que el desarrollo modificó por siempre el escenario mundial. Hasta ese momento, el término desarrollo era verificado solamente como una palabra sinónima de progreso, en la dirección de la escala peor para mejor, arcaico para actual, tradicional para más desarrollado o incluso del agrícola al urbano.

Actualmente, el desarrollo del término se utiliza de diversas formas, pudiendo también ser entendido y ser utilizado bajo conceptos diversos, desde el desarrollo económico, científico y tecnológico, social, el ser humano y el desarrollo local, así como otras denominaciones. Sin embargo, el término en sí, de acuerdo con los diccionarios de Michaelis en línea (2019) y Dicioline (2019), en cualesquiera de las concepciones se refiere a un acto, acción o efecto que está intrínsecamente conectado a un proceso de crecimiento o de progresión.

El desarrollo proporciona una evolución de lo que está puesto e independiente de las diversas concepciones en las que el término es utilizado, se nota que este proceso puede estar presente en objetos, lugares, personas, en alguna situación o incluso en una condición o fenómeno.

La palabra desarrollo siempre ha estado conectada a la cuestión del desarrollo económico, no obstante, para Sen (2005), nunca se debe percibir como siendo solamente económico, una vez que para él "[...] el desarrollo consiste en la eliminación de las privaciones de libertad que limitan las elecciones y las oportunidades de las personas de ejercer predominantemente su condición de agente" (SEN, 2005, p. 10).

Con una línea del pensamiento cercano a Sen, se verifica en Morin (2015, p. 27), que el desarrollo implica, por su vez, múltiplos desarrollos "de la prosperidad, del bienestar, de la mejora general de las condiciones de vida, de la reducción de las desigualdades, de la paz social y de la democracia". 
El autor también sostiene que la cuestión del desarrollo tiene lados tanto positivos como negativos; el lado positivo es que él enaltece algunas zonas de la prosperidad, observando la mejora de los padrones de vida, proporcionando autonomías individuales emancipadas con relación a la familia patriarcal, al final del casamiento impuesto, al brote de más libertad sexual y a las nuevas opciones del ocio. También proporciona el conocimiento de un mundo nuevo de la consumición, así como promueve las grandes aspiraciones democráticas.

Por otra perspectiva, Morin (2015), también señala lo que de negativo impone el desarrollo para la sociedad: la consumición del exacerbado, con un aumento del deseo por la adquisición de bienes y servicios, con nuevos deseos constantes, trae el individualismo, la incomprensión del otro y la búsqueda del beneficio cueste lo que cueste, puede crear nuevas corrupciones, con enormes zonas de miseria.

En este prisma, el desarrollo tendrá siempre dos lados a ser reflejados, no obstante, para reducir al mínimo lo negativo que se presenta, no se debe tener como concepto de desarrollo solo el lado económico, sino los múltiples desarrollos.

En este sentido, Vasconcellos y Garcia (1998, p. 205) afirman que debe haber la asignación de recursos "por los diversos sectores de la economía, con el objetivo de mejorar los indicadores del bienestar económico y social (pobreza, desempleo, desigualdad, condiciones de la salud, alimentación, educación y vivienda)". Ratifica Oliveira (2002, p. 1), al afirmar que “[...] el desarrollo, en cualquier concepto, debe resultar del crecimiento económico acompañado de la mejora en la calidad de la vida". Contemporáneamente, el concepto de desarrollo continúa conectado al desarrollo económico, no obstante, se incorporan a él otras dimensiones, considerando la libre elección individual y social, la efectiva participación en cuanto a tomar decisiones con respecto a la producción y distribución de riquezas y sus costes, sobre todo culturales y ambientales (BAQUERO; CREMONESE, 2008).

\subsubsection{Desarrollo económico}

Brasil, para Brum (2013, p. 545), es un país que en la vida de los brasileños aún se encuentra en construcción, pues "[...] en más de 500 años de historia oficial, aún no hemos logrado formar una nación con equilibrio social y vivenciar la participación de todos sus habitantes en un padrón y vida razonables".

El autor refuerza que existen enormes problemas históricos no resueltos, que se cumularon llevando a cuestiones de gran gravedad. Con esto, el país aun estando insertado en la realidad global de los tiempos actuales, un gran parte de la población no logra participar en dicha realidad. Según el censo (2010) el autor menciona que "[...] más de la mitad de 191 millones de brasileños (censo 2010) carece en cuestiones básicas, de una vida digna, como comer, vestirse, vivir, salud, escuela, cultura, trabajo, saneamiento básico, seguridad, ocio y otros" (BRUM, 2013, p. 545).

Los problemas históricos que no encontramos en Brasil, según Brum (2013), no pueden más ser pospuestos, pues un número muy grande de la población históricamente se encuentra en los bordes de la sociedad, viviendo en pobreza extrema y analfabetismo. Consecuentemente, mucho se tiene para hacer a fin de sanar los innumerables problemas históricos, sin embargo, se hace necesario proyectar el futuro del país, teniendo en cuenta un desarrollo económico con equidad, con la generación de la abundancia, del trabajo, de la renta y de las oportunidades, fortificando también la capacidad de investigación e innovación del país. De esta manera, para el 
autor, al verificar el desarrollo en el concepto económico, y aquí mencionando Brasil propiamente dicho, es necesario estar intrínsecamente volcado al desarrollo cultural, humano, local, etc.

El desarrollo económico es un proceso histórico en crecimiento, afirma Bresser-Pereira (2006), y se sostiene de la renta o de valor añadido por habitante, llevando a la población a una mejora en el estándar vivir, resultando, así, en una sistematizada acumulación de capital y de nuevos conocimientos que se incorporan junto al progreso técnico de la producción. Para el autor, el desarrollo económico ocurre por medio de un proceso de la transformación, el cual ira a alcanzar la sociedad de forma estructural, institucional o cultural, afirmando que:

[...] el aumento sostenido de los estándares de vida se ha hecho posible por el aumento de la productividad de determinadas actividades y/o por la transferencia de mano de obra de esas para otras actividades, con un valor agregado más grande per capita una vez que implican un más conocimiento. Es el aumento de los salarios causado directamente o indirectamente por ese incremento de la productividad y esa concentración de la población de un estadonación en las actividades de un valor agregado más grande. [...] el desarrollo económico promueve la mejora de los estándares de vida, pero no resuelve todos los problemas de una sociedad. Por lo tanto es solamente uno de los cinco grandes objetivos políticos a los que se proponen las sociedades modernas, al lado de la seguridad, de la libertad, de la justicia social y la protección del ambiente. (BRESSER-PEREIRA, 2006, p. 9).

El desarrollo económico es muy complejo, de hecho, es un desarrollo que por sí solo no resuelve los problemas de una sociedad a través de la generación de ingresos. También necesita brindar mayores oportunidades de acceso a bienes y servicios, a la educación, a la salud, a la vivienda, con el fin de promover otra concepción del desarrollo que es el desarrollo social.

Complementando esta afirmación, Souza (2007) afirma que el desarrollo económico es un crecimiento continuo que debe mejorar los indicadores económicos, sociales y ambientales. Es un fenómeno que se da a largo plazo, fortaleciendo la economía nacional, ampliando la economía de mercado, elevando la productividad del país y el nivel de bienestar de la población, con vistas a la preservación del medio ambiente. A través del desarrollo, "[...] la economía adquiere mayor estabilidad y diversificación, progreso tecnológico y formación de capital” (SOUZA, 2007, p. 7).

El autor también afirma que una definición más completa del término desarrollo incluye verificar la mejora de los indicadores económicos y sociales, observando una mayor productividad para el país junto con mejores niveles de bienestar de la población, para que el desarrollo económico no se produzca de forma aislada. Aún queda un tercer aspecto que no se puede olvidar, para que haya desarrollo económico: el tema del medio ambiente es fundamental. El tiempo hace con que el desarrollo económico agote o vuelva escasos los recursos naturales, si no hay un cuidado con el medio ambiente, no habrá recursos en el futuro, lo que afectará demasiadamente el desarrollo económico, o incluso, la vida en el planeta.

Desde esta perspectiva, se verifica Max-Neff (2007), en una entrevista realizada para la FNQ "Gestión para la transformación" ${ }^{4}$, en la que el autor menciona a los líderes empresariales, afirma que las empresas hoy tienen la obligación de ser conscientes de los problemas que involucran nuestro tiempo, "[...] Ante el drama que estamos viviendo hoy, todas y cada una de

\footnotetext{
${ }^{4}$ FNQ - Fundación Nacional de la Calidad - La "Fundação Nacional de Qualidade (FNQ)" es un centro brasileño de estudio, discute la irradiación del conocimiento sobre la excelencia en gestión. Creado en 1991, la FNQ es una institución sin ánimos de lucro, fundada por 39 organizaciones, privadas y públicas, cuyo objetivo es separar extensamente los Fundamentos y los Criterios de Excelencia en Gestión para las organizaciones de todos los sectores y portes. Disponible en: https://fnq.org.br/sobre-a-fnq/. Acceso el: 2 mayo 2019.
} 
las empresas deben trabajar de una manera que reduzcan los impactos negativos en el medio ambiente" (MAX-NEFF, 2007, p. 1), mencionando aún que las empresas ya no pueden esquivarse de esta responsabilidad.

El desarrollo económico ocurrirá siempre llevando en consideración otras dimensiones, observando: la calidad de vida de la población, la equidad social, la sustentabilidad del ambiente, el respecto a la cultura del pueblo, es decir, desarrollo económico debería caminar de manos dadas con el desarrollo de la sociedad.

\subsubsection{Desarrollo social}

El desarrollo social está en la evolución de la sociedad, en cuanto a la situación de la vida de la población, asegurando mejores oportunidades, la generación de renta, garantizando a la población el derecho a la educación, salud, vivienda y bienes y servicios.

El concepto de desarrollo social en América Latina surge entre las décadas de 1950 y 1960. El término ya tenía como presupuesto básico, según Rezende (2013, p. 94) de "[...] romper con las resistencias al cambio que fueron producidas por los parámetros estructurales (patrones de concentración de ingresos, de riqueza y de poder) vigentes en el continente".

El autor señala que el principal pensador de esta teoría en América Latina fue Celso Furtado. En sus diversos trabajos de 1992 a 2002, señaló que el desarrollo social estuvo en la transformación de elementos estructurales para el cambio de los patrones establecidos en la segunda mitad del siglo XX, que fue el tema de la desconcentración de la tierra y los ingresos.

En la década de los noventa la noción de desarrollo humano también tomó forma. Rezende (2013) sostiene que Celso Furtado en sus obras defiende una profunda transformación en la sociedad. Consolidando el concepto del desarrollo humano, aparece en esa época Sen Amartya, que converge en pensamiento con Celso Furtado al afirmar que:

Un concepto adecuado del desarrollo debe ir mucho más allá de la acumulación de la riqueza y del crecimiento del Producto Interior Bruto y de otras variables relacionadas con la renta. Sin ignorar la importancia del crecimiento económico, debemos mirar mucho más allá de él. [...] expandir las libertades que tenemos [...] no solo vuelve nuestra vida más rica y libre, sino que también nos permite ser seres sociales más completos, poniendo en práctica nuestras voliciones, interactuando con el mundo en que vivimos e influenciándolo. (SEN, 2005, p. 29).

Entonces se entiende que debe haber desarrollo económico sin que haya, paralelamente, el desarrollo social. No hay desarrollo donde la población se encuentra en la miseria, con hambre, sin educación y con enfermedades.

Es necesario que la población tenga acceso a todos sus derechos, para que haya equidad, promoción de la salud, el empleo y los ingresos y la educación. Si la sociedad está sana y goza de sus derechos, esto automáticamente se refleja en el desarrollo de la nación. El contrario también es un hecho, de esta manera donde no hay equilibrio entre lo económico y lo social, no hay un desarrollo efectivo.

\subsubsection{Capital humano y desarrollo}

El capital humano junto con el capital social forma parte del concepto de desarrollo social. Así, Max Neef (2012) afirma que el desarrollo social y personal es inseparable, para él una 
sociedad sana debería tener como regla el desarrollo de cada persona y de la persona entera. El autor, en una entrevista con la Fundación Nacional de la Calidad (FNQ) afirma que no cree en el crecimiento sino en el desarrollo, además de defender que la rentabilidad sin un enfoque en las personas no se sostiene (MAX-NEEF, 2007).

El Programa de las Naciones Unidas para el Desarrollo (PNUD) (BRASIL, 2019) menciona que el desarrollo humano es un proceso en el que las personas pueden ampliar sus opciones, aumentar sus capacidades y oportunidades, y pueden ser lo que quieren ser. También enfatiza que el desarrollo humano se diferencia del desarrollo económico, ya que busca ver a las personas en sus capacidades y oportunidades, mientras que el sesgo económico ve el bienestar de la sociedad a través de los recursos o ingresos que estas personas pueden generar.

La renta, sin duda alguna, es importante, pero debe ser percibida como una de las maneras y no el fin. "[...] es un cambio de perspectiva: con el desarrollo humano, el foco se transfiere del desarrollo económico, o de la renta, para el ser humano" (BRASIL, 2019, p. 1).

Para mensurar el desarrollo humano se utiliza el índice del desarrollo humano (IDH), que Ileva en cuenta las tres dimensiones del desarrollo: renta, educación y salud. El IDH apareció en 1990, una creación del Programa de las Naciones Unidas para el Desarrollo, contando con dos economistas, el paquistaní Haq de Mahbub UI Haq y el indio Amartya Sen. La creación del IDH tenía para el objetivo presentarse como:

[...] un contrapunto a otro indicador muy usado, el Producto Interior Bruto (PIB) per capita, que considera solamente la dimensión económica del desarrollo [...] el IDH se prepone ser una medida general y sintética que, aunque para ampliar la perspectiva en el desarrollo humano, no incluye tampoco agota todos los aspectos del desarrollo. (BRASIL, 2019, p. 1).

El IDH tiene como objetivo resaltar las brechas en las áreas de salud, educación y desigualdades sociales, ya que estos datos no son capturados por las cifras que aparecen en el PIB.

Amantya Sen, en una entrevista al programa "Memória Roda Viva", en 2001, hizo un comentario significativo sobre la importancia de la inversión social para el desarrollo, destacando el tema de la eliminación de la pobreza, mencionando su idea sobre la pobreza, que es mucho más que no tener unos ingresos bajos o nulos.

El vínculo entre la inversión social y la erradicación de la pobreza es muy fuerte. Funciona directa e indirectamente. La forma directa que quiero enfatizar es que cuando se genera más educación, más salud. Esto en sí mismo es una eliminación de la pobreza, porque la pobreza no es solo bajos ingresos, también es analfabetismo, también es no tener tratamiento médico cuando es necesario. Y al dar esto, se contribuye directamente a la eliminación de la pobreza. En segundo lugar, con la expansión de la educación y la mayor empleabilidad de personas con más educación, alfabetizadas en lugar de analfabetas, secundaria en lugar de primaria, una carrera en lugar de la secundaria, todos estos niveles, se puede incrementar la calidad de los recursos humanos involucrados. Está contribuyendo no solo al crecimiento económico, sino también a un sistema más compartido de crecimiento económico. Si las oportunidades económicas se comparten más, los frutos del crecimiento económico también lo serán. (SEN, 2001, p. 14).

El citado autor afirma que invertir en la solución de problemas sociales, en materia de salud, educación, ingresos y mejor equidad incidirá en el crecimiento económico, con vistas a un sistema más compartido.

Complementando a Sen, reitera Oliveira (2002), que para alcanzar el desarrollo humano se hace necesaria la reducción de la exclusión social, que es caracterizada por la desigualdad 
y la pobreza. También señala que la educación tiene un papel fundamental, ya que a través de ella la humanidad logrará dominar su propio desarrollo, dando la oportunidad a cada individuo de trazar su propio destino, contribuyendo responsablemente al progreso de la colectividad de la cual forma parte.

Amplían este pensamiento Borges y Bernartt (2011): para los autores, la enseñanza convencional no debe presentarse meramente como un proceso de contenidos desconectados en lo referente al hecho, sino como una forma de hacer con que el alumno conozca mejor la realidad donde está insertado y logre transformarla. No solo debe proporcionar conocimientos generales, sino que debe actuar para materializar estos conocimientos, con el fin de promover la mejora de la calidad de vida de las personas involucradas.

Respecto a esta formación, las autoras Borges y Bernartt (2011) afirman que la escuela no puede ser un mero proceso de adquisición de contenidos, sino que tiene un papel importante en cuanto al proceso de articulación entre las necesidades del desarrollo local y los conocimientos transmitidos a los estudiantes. Estos deben configurarse como instrumentos de intervención sobre una realidad dada, permitiendo que todos tengan iniciativas más inteligentes y conscientes, y también se sugieren posibilidades para el desarrollo humano.

\subsubsection{Desarrollo local}

El desarrollo local puede entenderse como las fuerzas sociales, económicas, culturales y políticas que actúan en un territorio determinado, con características propias y reflejando cambios cualitativos en la estructura de la localidad.

En palabras de Ávila el desarrollo local se puede definir como siendo:

[...] fundamentalmente en el desarrollo efectivo de las capacidades, competencias y habilidades de una "comunidad definida" (por tanto, con intereses comunes y ubicada en un determinado territorio o lugar con identidad social e histórica), en el sentido de ella misma volverse paulatinamente apta a agenciar y gestionar (diagnosticar, tomar decisiones, planificar, actuar, evaluar, controlar, etc.) el uso de sus propios potenciales, así como la "metabolización" comunitaria de insumos e inversiones públicas y privadas externas, con vistas a la búsqueda procedimental de soluciones a los problemas, necesidades y aspiraciones, de todo orden y naturaleza, que le conciernen más directa y cotidianamente. (ÁVILA, 2000, p. 68).

El desarrollo local se da de adentro hacia afuera, es decir, revisando los problemas locales, los intereses comunes en la comunidad, el potencial de la región, y con base en el conocimiento existente en la zona, la comunidad comienza a dar sus propias respuestas, ya sea para diagnosticados. problemas $u$ otras aspiraciones. Puede verse como un proceso continuo, donde la propia comunidad, al ser consciente de sus problemas y aspiraciones, a través de esfuerzos sucesivos, busca en sus potencialidades, competencias y habilidades, soluciones locales a los problemas e inquietudes detectadas.

Milani (2004) corrobora este pensamiento, ya que, para él, el desarrollo local puede ser percibido como un conjunto de actividades entre los diferentes elementos que componen una sociedad como su cultura, economía, medio ambiente, política, educación y tecnología, así como agentes que están presentes en diferentes escalas de la economía y la política, es decir, de lo local a lo global.

Valorar solo a los agentes económicos como actores del desarrollo es una vieja tendencia, olvidar a la sociedad y a los grupos excluidos como motores del desarrollo. Hablar de desarrollo 
local es recordar que todos los involucrados son elementos indispensables. "[...] Es decir, todos los actores sociales públicos y privados que están presentes en un determinado espacio geográfico, deben ser movilizados para participar en el proceso. Todos sin excepción son considerados piezas importantes del tablero" ${ }^{5}$ (COUTO, 2006, p. 4 apud Oliveira, 2013, p. 57).

Para que la comunidad aproveche el potencial local, debe desarrollar los conocimientos existentes, esto ocurre a través de la búsqueda de la formación y educación en cuanto a las habilidades y habilidades necesarias para el desarrollo de dicha potencialidad.

[...] el desarrollo local implica la formación y educación de la propia comunidad en cuanto a cultura, capacidades, habilidades y destrezas que le permitan, por supuesto, con la ayuda de todos los agentes y factores externos, gestionar y gestionar el conjunto del proceso de desarrollo de la respectiva localidad, en lugar de simplemente participar en propuestas o iniciativas que vienen de fuera. (BROSTOLIN, 2007, p. 108).

La educación cambia la vida de las personas, para Oliveira (2013) es un factor en el aumento de la productividad de las empresas, resultando en una mayor competitividad de la población en el escenario económico, y al incrementar su producción, transcurre en posibilidad de progreso tecnológico y competitividad para las personas. Borges y Bernartt (2011, p. 56) complementan, "[...] la educación fortalece y difunde valores, principios y normas de convivencia construidas colectivamente y transmitidas por la situación cotidiana, fortaleciendo los lazos de pertenencia".

La educación y la formación se entrelazan, que para Ávila (2000) es a través de la educación que el niño o cualquier otro alumno puede desarrollar sus capacidades físicas, morales, intelectuales, así como sociales. Consiste en una forma de pasar de un estado básico a otro con mayor mejora. La formación, en cambio, "se sitúa en un nivel básico de búsqueda, desciframiento, discernimiento e incorporación de significados y valores de una determinada realidad" (ÁVILA, 2000, p. 63).

Ávila (2000), aún señala que la educación y la formación se complementan, ya que la educación implica una formación con fundamento, y la formación está en la necesidad de la educación para implementar la dinámica de la vida del ser humano. Así, se entiende que la comunidad deberá estar en constante formación, utilizando la educación formal que recibe en las escuelas y la no formal, que adquiere empíricamente, es decir, a partir de los conocimientos vividos que se encuentran en la experiencia acumulada de cada localidad.

El Corredor Bioceánico, como corredor de desarrollo, trae perspectivas de expansión de nuevos horizontes a los puntos de cruce, y en este primer momento, con el inicio de las actividades del Corredor Vial, existe la necesidad de formación del capital humano, para que estas perspectivas sean realmente efectivas.

\section{CAPACITACIÓN Y CALIFICACIÓN DEL CAPITAL HUMANO CON VISTAS A LA IMPLEMENTACIÓN DEL CORREDOR BIOCEÁNICO}

La nueva realidad que el Corredor Bioceánico promete al Estado de Mato Grosso do Sul trae excelentes perspectivas, sin embargo, juntos surgen cuestiones urgentes que deben ser trabajadas para que el éxito del proyecto se convierta en una realidad. Además de la infraestructura, los

\footnotetext{
${ }^{5}$ Y afirmar que todos los actores sociales públicos o privados que están presentes en determinados espacios geográficos, el territorio debe ser movilizado para participar en el proceso. Todos, sin excepción, se consideran piezas importantes del consejo.
} 
temas legales, aduaneros, logísticos, entre otros, un punto de suma importancia es la formación de capital humano.

El trabajo, según Manfredi (2002, p. 33) “[...] constituye una base fundacional de la economía de cualquier sociedad, una fuerza social para la producción de bienes y servicios y una fuente de ingresos y supervivencia para grandes segmentos de la población humana". De esta manera, se ampliará el trabajo tanto formal como informal para atender las nuevas demandas locales con el inicio de actividades en el Corredor Bioceánico y consecuentemente de la Ruta con sus perspectivas culturales, sociales y turísticas. En este contexto, existen importantes proyecciones de nuevos puestos de trabajo, que requieren la formación, calificación y recalificación de la mano de obra.

En este contexto, el capital humano surge de la cuestión de la importancia de la educación en la formación de personas más productivas y en consecuencia apunta a las siguientes reflexiones: ¿cuál es la relación entre crecimiento económico, educación y capital humano? ¿Hay posibilidades de que el capital humano influya en el desempeño de una región?

Considerado el precursor de la teoría del capital humano, Mincer (1958) ya señaló la correlación existente entre la inversión en la formación de personas (trabajadores) con la distribución del ingreso personal, enfatizando así la necesidad de obtener nuevos conocimientos en las actividades profesionales y no en el mantenimiento en el trabajo sin posibilidades de formación y estudios en busca de nuevos conocimientos.

Schultz (1964) refuerza este pensamiento, cuando afirma la importancia de la inversión en educación en la calificación y mejora de la población con vistas a la productividad, consecuentemente al impacto de la economía.

Ampliando esta discusión, Kliksberg (1999) destaca así que el capital humano y el capital social han obtenido una participación mayoritaria en el desempeño económico regional, siendo que el primero puede ser caracterizado por los niveles de nutrición, educación y salud de la población y capital social por la posibilidad de asociación entre los individuos como una forma de crecimiento económico tanto local como regional.

Pensando no solo en la mejora del nivel educativo sino también en las competencias de los trabajadores, a partir de la formación, los conocimientos específicos, Almeida y Pereira (2000) destacan que estas acciones son factores importantes para el crecimiento económico. Otro aspecto planteado por los autores se refiere a la importancia de insertar capital humano en el espacio geográfico tanto para reducir las desigualdades sociales como también como un dispositivo que puede paliar las disparidades regionales, basado en el crecimiento y el desarrollo socioeconómico entre regiones.

Las políticas de desarrollo son factores que brindan el incremento de nuevos puestos de trabajo, según Manfredi (2002, p. 50), “[...] mecanismos de crecimiento económico, como políticas de desarrollo, creación de nuevos puestos de trabajo, distribución de renta (entre otros), es que son responsables por crear nuevos puestos de trabajo e incluso nuevas ocupaciones".

Para la SEMAGRO (2020), la Ruta Bioceánica es un proyecto estratégico nacional, según la posición del senador por Mato Grosso do Sul, Nelson Trad "La Ruta Bioceánica promoverá un incremento en el desarrollo de las ciudades por donde pase. Se trata de un cambio positivo en los escenarios socioeconómicos de estas localidades" (SEMAGRO, 2020, p. 1). Esto requiere una planificación y preparación para que el proyecto sea efectivo, no solo con la posibilidad de generar empleos e ingresos, sino también con el desarrollo de las localidades, utilizando mano de obra local. 
Un punto observado por Gary Becker, premio Nobel de Economía en 1992, señala que el diferencial de las empresas no está solo en el avance tecnológico, que rápïdamente es copiado por la competencia, sino en la calidad de los servicios prestados. Afirmaba que el éxito de las personas que brindan servicios está cada vez más ligado a la educación, para él, según Kelniar, Lopes y Pontili (2013, p. 4) "la inversión en educación contribuye al crecimiento económico, mejora los ingresos individuales y tiene un impacto positivo efecto sobre la salud y la formación de las familias".

Así, en lo que respecta a la preparación del capital humano que será necesario para el frente de trabajo en esta nueva perspectiva de Mato Grosso do Sul, son muchos los cursos de calificación y recalificación que podrán ofrecer las instituciones educativas. Estos cursos pueden ser ofrecidos en forma de titulaciones rápidas, cursos de formación inicial y continua, atendiendo a diversos ejes como Infraestructura, Turismo, Hotelería y Ocio, Gestión y Negocios o incluso mediante formación técnica, presencial y a distancia. Modalidades de aprendizaje, que pueden ser cursos técnicos integrados, posteriores o concomitantes, eso dependerá de la realidad de cada municipio.

\subsection{Cursos de formación inicial y continuada}

En cuanto a los cursos rápidos que pueden ser ofrecidos por instituciones educativas calificadas, actualmente, como guía para estos cursos, la Guía Pronatec, edición 2016, un documento que enumera la educación inicial y continuada (FIC) o cursos de calificación profesional, propone cursos con una carga de trabajo de al menos 160 horas y organizados en 12 ejes tecnológicos, son 646 sugerencias de cursos apuntando al eje al que pertenece, la formación requerida, el perfil profesional, la edad para realizar el curso y las Ocupaciones Asociadas (CBO). La última actualización de este catálogo realizada por el MEC es de 2016, sin embargo, las instituciones oferentes lo utilizan como guía.

Así, para demostrar posibilidades de ofrecer cursos rápidos a los municipios que se encuentran dentro del recorrido de la Ruta Vial Bioceánica, existen algunos cursos que pueden colaborar en el corto, mediano y largo plazo para la necesaria calificación de los recursos humanos disponibles en los municipios donde la Ruta pasará, impidiendo la búsqueda en otras ciudades, o incluso en otros Estados, de la mano de obra necesaria.

La formación condecente deberá partir de cada localidad, una vez que las realidades son diferentes, debiendo cada gobierno local realizar el análisis de las necesidades y buscar la asociación con las instituciones de educación, a fin de proveer a su población de la atención a sus carencias laborales. A continuación, a modo de explicación de las posibilidades de formaciones que atienden a las ciudades por donde pasará la Ruta Vial Bioceánica, insertamos la cuadro 1:

Cuadro 1 - Posibilidades de cursos rápidos

\begin{tabular}{|l|l|c|}
\hline \multicolumn{1}{|c|}{ Curso } & \multicolumn{1}{c|}{ Eje Tecnológico } & Horas de Crédito \\
\hline Agente de Aeropuerto & Infraestructura & $200 \mathrm{~h}$ \\
\hline Agente de Estación de Trenes & Infraestructura & $400 \mathrm{~h}$ \\
\hline Agente de Informaciones Turísticas & Información y Comunicación & $200 \mathrm{~h}$ \\
\hline Arreglador y Verificador de Cargas & Infraestructura & $160 \mathrm{~h}$ \\
\hline Asistente de logística & Gestión y Negocios & $160 \mathrm{~h}$ \\
\hline Asistente de Operaciones de Logística de Puertos & Infraestructura & $160 \mathrm{~h}$ \\
\hline
\end{tabular}




\begin{tabular}{|l|l|c|}
\hline \multicolumn{1}{|c|}{ Curso } & \multicolumn{1}{c|}{ Eje Tecnológico } & Horas de Crédito \\
\hline Asistente de Servicios en Comercio Exterior & Gestión y Negocios & $160 \mathrm{~h}$ \\
\hline Dependiente de Cafetería & Turismo, Hospitalidad y Ocio & $160 \mathrm{~h}$ \\
\hline $\begin{array}{l}\text { Auxiliar de Transporte, Desplazamiento y } \\
\text { Distribución de Cargas }\end{array}$ & Infraestructura & $240 \mathrm{~h}$ \\
\hline Camarera en Medios de Alojamiento & Turismo, Hospitalidad y Ocio & $200 \mathrm{~h}$ \\
\hline Conductor de Turismo de Aventura & Turismo, Hospitalidad y Ocio & $240 \mathrm{~h}$ \\
\hline Conductor de Turismo de Pesca & Turismo, Hospitalidad y Ocio & 160 \\
\hline $\begin{array}{l}\text { Conductor de Turismo en Espacios Culturales } \\
\text { Locales }\end{array}$ & Turismo, Hospitalidad y Ocio & $200 \mathrm{~h}$ \\
\hline $\begin{array}{l}\text { Conductor de Turismo en Unidades de } \\
\text { Conservación Ambiente Local }\end{array}$ & Turismo, Hospitalidad y Ocio & $200 \mathrm{~h}$ \\
\hline Español aplicado a los Servicios Turísticos & Turismo, Hospitalidad y Ocio & $180 \mathrm{~h}$ \\
\hline Mesero & Turismo, Hospitalidad y Ocio & $200 \mathrm{~h}$ \\
\hline Inglés aplicado a los Servicios Turísticos & Turismo, Hospitalidad y Ocio & $180 \mathrm{~h}$ \\
\hline Operador de movimiento de cargas & Infraestructura & $260 \mathrm{~h}$ \\
\hline Albañilería Estructural & Infraestructura & $160 \mathrm{~h}$ \\
\hline Recepcionista & Gestión y Negocios & $160 \mathrm{~h}$ \\
\hline Supervisor de Transportes Terrestres & Infraestructura & $240 \mathrm{~h}$ \\
\hline
\end{tabular}

Fuente: Elaborado por los autores- Basado en la Guía Pronatec (BRASIL, 2016).

Este cuadro llevó en cuenta algunos cursos incluidos en la Guía Pronatec, sin embargo, este estudio solo pretende demostrar algunas posibilidades de formación, por lo que los municipios podrán ampliar el tema del estudio de idiomas, así como la formación dirigida a la artesanía de cada localidad, entre muchas otras que pueden ser imperativas.

\subsection{Cursos Técnicos}

En relación a los cursos técnicos, se pueden ofrecer tanto en metodología presencial como a distancia, con tres posibilidades, el técnico integrado, sucesivo o concomitante. El curso técnico integrado se trata de la circunstancia en que la educación secundaria se realiza junto con la técnica, para que el alumno pueda realizarla, debe haber completado la educación primaria (INSTITUTO FEDERAL DO RIO GRANDE DO SUL, 2021). En Mato Grosso do Sul son pocos los municipios que cuentan con este tipo de oferta, que se realiza de forma presencial. En cuanto al curso técnico sucesivo, el alumno deberá haber completado la secundaria, ya que solo realizará la parte que pertenece a la Educación Técnica (IFRS, 2021).

Son muchas las instituciones que trabajan el técnico sucesivo a distancia. Según Oliveira (2013), el Instituto Federal de Mato Grosso do Sul tiene un historial de alianzas con Ayuntamientos para llevar a cabo esta modalidad a distancia. Finalmente, el técnico concomitante también existe, de tal manera que el aprendiz atiende a una educación media del curso en una institución y en el otro turno realiza la educación técnica del área de interés (IFRS, 2021), que puede ser tanto en persona como a distancia.

A modo de verificación de ofertas de cursos técnicos que pueden colaborar con la formación laboral para los municipios de Mato Grosso do Sul por donde pasará la Ruta Vial Bioceánica, a continuación, constan cursos del Catálogo Nacional de Cursos Técnicos, actualizado en 2021. "El Catálogo Nacional de Cursos Técnicos (CNCT) disciplina la oferta de cursos de educación 
técnica profesional de nivel secundario para orientar e informar a las instituciones educativas, estudiantes, empresas y a la sociedad en general" (BRASIL, 2021, p. 17).

El catálogo subvenciona a instituciones que ofrecen este tipo de modalidad de la educación, cuenta con 13 ejes y provee subsidios para 215 cursos, indicando la carga mínima del horario, el perfil profesional de conclusión, infraestructura mínima requerida, entre otras informaciones pertinentes. El Cuadro 3, según el catálogo de cursos (BRASIL, 2021), enumera algunos cursos que podrían colaborar con los municipios en este momento de formación:

Cuadro 2 - Posibilidades de cursos técnicos

\begin{tabular}{|l|l|c|}
\hline \multicolumn{1}{|c|}{ Curso } & \multicolumn{1}{c|}{ Eje } & $\begin{array}{c}\text { Crédito de } \\
\text { horas mínimo } \\
1200 \mathrm{~h}\end{array}$ \\
\hline Técnico Mecánico & Control de procesos industriales & $800 \mathrm{~h}$ \\
\hline Técnico en Comercio & Gestión y Negocios & $800 \mathrm{~h}$ \\
\hline Técnico de Comercio Exterior & Gestión y Negocios & $800 \mathrm{~h}$ \\
\hline Técnico en Logística & Gestión y Negocios & $800 \mathrm{~h}$ \\
\hline Técnico en Ventas & Gestión y Negocios & $1200 \mathrm{~h}$ \\
\hline Técnico en Informática & Información y Comunicación & $1000 \mathrm{~h}$ \\
\hline Técnico en Carreteras & Infraestructura & $1000 \mathrm{~h}$ \\
\hline Técnico en Puertos & Infraestructura & $800 \mathrm{~h}$ \\
\hline Técnico en Transporte de Cargas & Infraestructura & $800 \mathrm{~h}$ \\
\hline Técnico en Agencia de Viajes & Turismo, Hospitalidad y Ocio & $800 \mathrm{~h}$ \\
\hline Técnico en Guía Turístico & Turismo, Hospitalidad y Ocio & $800 \mathrm{~h}$ \\
\hline Técnico en Alojamiento & Turismo, Hospitalidad y Ocio & \\
\hline Técnico en Servicios de Bares y Restauración & Turismo, Hospitalidad y Ocio & \\
\hline
\end{tabular}

Fuente: Elaborado por los autores en base al Catálogo Nacional de Cursos Técnicos (2021).

Existen numerosas posibilidades de formación, tanto desde cursos de formación inicial como continuada, considerados cursos rápidos, así como cursos de formación técnica, siendo cada municipio analizado, ya que los cursos, subvenciones y asociaciones para su realización serán responsabilidad del poder público de cada localidad.

\section{CONSIDERACIONES FINALES}

La ruta bioceánica que integra Brasil, Paraguay, Argentina y Chile, como corredor de exportaciones e importaciones, trae la promesa de desarrollo, en sus diversas concepciones, al Estado de Mato Grosso do Sul, con perspectivas de expansión y cambio en la economía. del estado. Existen innumerables posibilidades, ya que, históricamente, las principales actividades económicas del estado siempre han estado ligadas a la agricultura, la ganadería y la agroindustria, con la implementación de la ruta bioceánica, una nueva realidad se vislumbra. El Estado cuenta ahora con un nuevo mercado por agregar y explorar, que es el negocio de exportación, ya que será parte del camino a realizar hasta los puertos de Chile en el Océano Pacífico, con el objetivo de acceder a las exportaciones a los mercados asiáticos, de Oceanía, de América del Norte, así como para países de América del Sur.

Para que el corredor Bioceánico sea efectivo existe la necesidad de operaciones multimodales, es decir, el uso de los transportes viales, hechos por ríos, aéreos y de ferrocarril, lo que deberá ocurrir en el transcurso de los años, sin embargo, al principio los gobiernos de 
los países implicados (Brasil, Paraguay, la Argentina y Chile) están enfocando sus esfuerzos en el transporte de vial.

El Corredor Bioceánico no solo se presenta como un pasaje para el transporte de mercancías, sino que la ambición es que sea un corredor de desarrollo, ya que las localidades que se encuentren en su camino deberán prepararse para las nuevas demandas que se avecinan. Al principio, pasará para esas localidades que las flotas de los camiones con mercancías que están de camino a los puertos de Chile, las ciudades tendrán que mejorar sus estructuras, ampliando servicios en áreas diversas, lo que resulta en nuevas empresas o bien en la ampliación de las ya existentes.

Junto con el corredor de exportación, las expectativas para el turismo son grandes. Es sabido que habrá la ampliación del flujo turístico a fin de conocer las bellezas del Pantanal de Mato Grosso do Sul, así como los encantos del pueblo de Bonito y demás pueblos que ser presentan con potencialidad para el ecoturismo, y seguir conociendo los atractivos turísticos de los demás países que la ruta compone.

La cultura de las localidades, sus artesanías, monumentos, música, arte e historias, con el aumento del flujo turístico o incluso de un flujo más grande de personas a pasar por estas localidades, conocidas y compartidas entre los países que componen la ruta.

Muchas inversiones has sido realizadas por el poder público para atender la infraestructura necesaria, buscando la ampliación de las vías de transporte, cuestiones vinculadas al análisis de los impactos ambientales, trámites con los países a lo que se refiere a cuestiones jurídicas y aduaneras, además de trámites para los puertos secos, etc., no obstante, se nota que aún hay mucho que hacer.

El hecho es que las localidades tendrán que adaptarse a estas nuevas demandas, ampliando sus redes de servicios y mejorando sus atendimientos, así como nuevos puestos de trabajo con el surgimiento de profesiones que hasta entonces no había en nuestro estado. En este contexto, existe la necesidad de formación, calificación y recalificación del capital humano. Las empresas y los poderes públicos deben mirar hacia el futuro y preparar a las personas con la formación adecuada, evitando que se busque mano de obra preparada en otras ciudades o incluso estados.

Así, este estudio procuró evidenciar las preguntas que implican los cambios que el estado de Mato Grosso do Sul está por vivir con la llegada de una ruta de exportación y traer a la tona la importancia de la cuestión de la formación del capital humano. Exponer, también, como sugerencia, diversos cursos que podrán ser desarrollados en asociaciones del poder público local, empresas e instituciones de enseñanza, para que formación local se dé, haciendo con que realmente se concretice el desarrollo en sus diversos conceptos, de las comunidades involucradas en el Corredor.

\section{REFERENCIAS}

ALMEIDA, E. P.; PEREIRA, R. S. Críticas à teoria do capital humano: uma contribuição à análise de políticas públicas em educação. Revista de Educação Pública, Cuiabá, v. 9, n. 15, p. 53-70, jun. 2000.

ÁVILA, Vicente Fideles. Pressupostos para formação educacional em desenvolvimento local. Interações, Campo Grande, MS, v. 1, n. 1, p. 63-76, 2000. Disponible en: http://www.interacoes.ucdb.br/article/ view/616/640. Acceso el: 30 abril 2018

BAQUERO, Marcello; CREMONESE, Dejalma (Org.). Desenvolvimento regional, capital social e democracia local. Ijuí: Unijuí, 2008.

INTERAÇÕES, Campo Grande, MS, v. 22, n. 4, p. 1113-1131, out./dez. 2021. 
BARROS, Pedro Silva; PADULA, Raphael; SEVERO, Luciano Wexell; SAMURIO, Sofía Escobar; JULIA, Souza Borba Gonçalves. Corredor Bioceânico de Mato Grosso do Sul ao pacífico: produção e comércio na rota de integração sul-americana. Campo Grande: UEMS; Brasília, DF: Ipea, 2020.

BRASIL. Ministerio de Educación. Departamento de Educación Profesional y Tecnológica. Guía Pronatec. Brasília-DF, 2016. Disponible en: https://map.mec.gov.br/projects/parceiros-demandantes-e-ofertantesda-bolsa-formacao/wiki/Guia_FIC_-_4\%C2\% AA_Edi\% C3\% A7\% C3\% A3o. Acceso el: 27 mayo 2021.

BRASIL. Ministerio de Educación Departamento de Formación Profesional y Tecnológica. Catálogo de Cursos Técnicos - CNCT. 4. ed. Brasília-DF: MEC, 2021. Disponible en: http://cnct.mec.gov.br/cnct-api/ catalogopdf. Acceso el: 27 mayo 2021.

BRASIL. Programa de las Naciones Unidas para el Desarrollo. Desarrollo humano e IDH. Brasília-DF: PNUD, 2019. Disponible en: http://www.br.undp.org/content/brazil/pt/home/idh0.html. Acceso el: 12 jun. 2021.

BENITES, Súzan. Rota Bioceânica tem potencial para movimentar $R \$ 1,5$ bilhão em Mato Grosso do Sul. Correio do Estado, Campo Grande, MS, 21 nov. 2020. Disponible en: https://correiodoestado.com.br/ economia/rota-bioceanica-tem-potencial-para-movimentar-r-15-bilhao/379613. Acceso el: 13 mayo 2021.

BORGES Graziela Scopel; BERNARTT, Maria de Lourdes. Educação e desenvolvimento local. Mexico Documents, 2011. Disponible en: https://vdocuments.mx/educacao-e-desenvolvimento-local.html. Acceso el: 12 jul. 2021.

BRESSER-PEREIRA, Luiz Carlos. O conceito histórico de desenvolvimento econômico. Trabalho originalmente preparado para curso de Desenvolvimento Econômico na Fundação Getúlio Vargas. Versão de 2 de março de 2006. Disponible en: http://bresserpereira.org.br/papers/2006/06.7-conceitohistoricodesenvolvimento. pdf. Acceso el: 23 jul.2021.

BROSTOLIN, Marta Regina. Educação e desenvolvimento: uma parceria necessária na construção da emancipação sociocultural das populações indígenas. Revista Tellus, Campo Grande, MS, año 7, n. 12, p. 103-14, abr. 2007. Disponible en: http://www.tellus.ucdb.br/index.php/tellus/article/viewFile/132/138. Acceso el: 10 jul. 2021.

BRUM, Argemiro Jacob. O desenvolvimento econômico brasileiro. 30. ed. Petrópolis: Vozes; ljuí: Unijuí, 2013.

CASTRO, João Carlos Parkinson. Turismo como instrumento dinamizador do Corredor Rodoviário Bioceânico. Interações, Campo Grande, v. 20, n. especial, 2019. Disponible en: https://interacoesucdb. emnuvens.com.br/interacoes/article/view/2419. Acceso el: 2 marzo 2021.

CONSTANTINO, M.; DORSA, A. C.; ARAGÃO, J. C. M.; MENDES, D. R. F. Fluxos turísticos entre os países do Corredor Bioceânico. Interações, Campo Grande, MS, v. 20(especial), p. 57-67, 2019. Disponible en: https://doi.org/10.20435/inter.v20iespecial. Acceso el: 2 mayo 2021.

DICIO-ONLINE. Dicionário on-line da língua portuguesas. Desenvolvimento. Disponible en: https://www. dicio.com.br/desenvolvimento. Acceso el: 3 jul. 2021.

FUNDAÇÃO NACIONAL DA QUALIDADE [FNQ]. História da FNQ: há três décadas dedicada à excelência da gestão. FNQ, São Paulo, 2020. Disponible en: https://fnq.org.br/sobre-a-fnq/. Acceso el: 2 mayo 2021.

INSTITUTO FEDERAL DO RIO GRANDE DO SUL. Cursos. 2021. Disponible en: https://ifrs.edu.br/cursos/. Acceso el: 20 jun. 2021. 
KELNIAR, V. C.; LOPES, J. L.; PONTILI, R. M. A Teoria do Capital Humano: revisitando conceitos. In: ENCONTRO DE PRODUÇÃO CIENTÍFICA E TECNOLÓGICA, 8., 21 a 25 oct. Campo Mourão, PR. Anais [...]. Campo Mourão: UNESPAR/NUPEM, 2013. Disponible en http://www.fecilcam.br/nupem/anais_viii_epct/PDF/TRABALHOSCOMPLETO/Anais-CSA/ECONOMICAS/05-Vckelniartrabalhocompleto.pdf . Acceso el: 13 mayo 2021.

KLIKSBERG, B. Capital social y cultura, claves esenciales del desarrollo. Revista de la CEPAL, Santiago de Chile, n. 69, 1999.

MANFREDI, Sílvia Maria. Educação profissional no Brasil. São Paulo: Cortez, 2002.

MATO GROSSO DO SUL (Estado). Investimentos do Estado em logística em Murtinho avançam fronteira agrícola no Sudoeste. Portal del Gobierno de Mato Grosso do Sul (15/02/21) 2021a Disponible en: http:// www.ms.gov.br/investimentos-do-estado-em-logistica-em-murtinho-avancam-fronteira-agricola-nosudoeste/. Acceso el 3 de jun.2021.

MATO GROSSO DO SUL. Secretaria de Estado de Meio Ambiente, Desenvolvimento Econômico, Produção e Agricultura Familiar. Implantação da Rota Bioceânica é projeto estratégico nacional, afirma secretario. SEMAGRO, Campo Grande, MS, 24 agosto 20. Disponible en: https://www.semagro.ms.gov.br/implantacaoda-rota-bioceanica-e-projeto-estrategico-nacional-afirma-secretario/. Acceso el: 10 mayo 2021.

MAX-NEEF, Mandref. Desenvolvimento à escala humana: concepção, aplicação e reflexões posteriores. Blumenau: EDIFURB, 2012.

MAX-NEEF, Manfred. O economista chileno Manfred Max-Neef contrapõe crescimento econômico e qualidade de vida. Entrevista el 17 abr. 2007. Disponible en: http://www.parceirosvoluntarios.org.br/oeconomista-chileno-manfred-max-neef-contrapoe-crescimento-economico-e-qualidade-de-vida/. Acceso el: 3 de abr. 2018.

MICHAELIS. Diccionario on-line de la lengua portuguesa. Desenvolvimento. 2019. Disponible en: http:// michaelis.uol.com.br. Acceso el: 3 de febrero. 2019.

MILANI, Carlos. Teorias do capital social e desenvolvimento local: lições a partir da experiência de Pintadas, Bahia, Brasil. Revista O\&S, Salvador, v. 11, edición especial, p. 95-113, 2004. Disponible en: https:// portalseer.ufba.br/index.php/revistaoes/article/view/12637/8906. Acceso el: 8 agosto 2018.

MINCER, Jacob. Investment in human capital and personal income distribution. Journal of Political Economy, Chicago, v. 66, n. 4, p. 281-302, 1958.

MORIN, Edgar. O Método III - o conhecimento do conhecimento. Porto Alegre: Sulina, 2015

OLIVEIRA, Edilene Maria. A educação a distância do Instituto Federal de Educação, Ciência e Tecnologia de Mato Grosso do Sul: perspectivas para o desenvolvimento local. 2013. 128 f. Tesis (Máster en Desarrollo Local) - Universidade Católica Dom Bosco, Campo Grande, MS, 2013.

OLIVEIRA, Gilson Batista. Uma discussão sobre o conceito de desenvolvimento. Revista FAE, Curitiba, v. 5, n. 2, p. 37-48, 2002. Disponible en: https://revistafae.fae.edu/revistafae/article/view/477. Acceso el: 20 enero 2021.

REZENDE, Maria José. As noções de desenvolvimento social como base para a abordagem do desenvolvimento humano presente no Relatório do Desenvolvimento Humano (RDH) de 1990. Revista Ensaios FEE, Porto Alegre, v. 34, n. 1, p. 91-122, 2013. Disponible en: https://revistas.fee.tche.br/index. php/ensaios/article/download/2535/3151. Acceso el: 1으 mayo 2021. 
SEN, Amarthya. Memória Roda Vida. Entrevistada por. São Paulo: Entrevista concedida a M. Teixeira, Programa Roda Vida de TV Cultura, Fundación Padre Anchieta-Centro São Paulo de Radio y TV Educativas, em 22 enero 2001. Disponible en: . Acceso el: 6 de abril. 2021.

SEN, Amarthya. Desenvolvimento como liberdade. São Paulo: Cia das Letras, 2010.

SOUZA, Nali de Jesús. Desenvolvimento econômico. 5. ed. reimpr. São Paulo: Atlas, 2007.

SCHULTZ, Theodore William. O valor econômico da educação. Rio de Janeiro: Zahar Editores, 1964.

VASCONCELLOS, Marco Antonio; GARCIA, Manuel Enriquez. Fundamentos de economia. São Paulo: Saraiva, 1998.

VIEGAS, A. Com rota bioceânica e três novos portos, cidade de MS deve se transformar em 'hub logístico' do país. G1 MS, Campo Grande, MS, 20 jul. 2019. Disponible en https://g1.globo.com/ms/mato-grossodo-sul/noticia/2019/07/20/com-rota-bioceanica-e-tres-novos-portos-cidade-de-ms-deve-se-transformarem-hub-logistico-do-pais.ghtml. Acceso el: 3 abr. 2021.

\section{Sobre las autoras y el autor:}

Edilene Maria de Oliveira: Doctorado y Máster en Desarrollo Local por la Universidad Católica Dom Bosco (UCDB). Especialización en Administración, Marketing y Comercio Exterior por la UCDB; especialización en Educación de Jóvenes y Adultos por la Universidad de Brasilia (UNB); especialización en Gestión de Iniciativas Sociales por la Universidad Federal de Río de Janeiro (UFRJ); y especialización en Docencia por el Instituto Federal de Mato Grosso do Sul (IFMS). Grado en Trabajo Social por las Facultades Unidas Católicas de Mato Grosso (FUCMT) y en Administración Pública por la UCDB. Profesora de Educación Técnica y Tecnológica en Administración, en IFMS. E-mail: edilene.oliveira@ifms.edu.br, Orcid: http://orcid.org/0000-0002-7787-9012

Arlinda Cantero Dorsa: Realizando prácticas postdoctorales en Desarrollo Local en el Centro Universitario Augusto Motta (UNISUAM). Doctorado en Lengua Portuguesa por la Pontificia Universidad Católica de São Paulo (PUC-SP). Máster en Comunicación y Letras por la Universidad Presbiteriana Mackenzie (UPM). Grado en Letras/Francés por la Facultad Dom Aquino de Filosofía, Ciencias y Letras; y en Pedagogía por la Facultad de Educación, Ciencias y Letras de Urubupungá (FECLU). Profesora titular de la Universidad Católica Dom Bosco (UCDB), en el curso de Derecho. Profesora y vicecoordinadora del Máster y Doctorado en Desarrollo Local de la UCDB. Miembro del Instituto Histórico e Geográfico de Mato Grosso do Sul (IHG-MS). Líder del Grupo de Investigación en Patrimonio Cultural, Derecho y Diversidades y subdirectora del Grupo de Investigación y Estudios en Tecnología Educativa y Educación a Distancia (GETED) de la UCDB. Coordina el proyecto Ruta Bioceánica y UNIRILA (Red Universitaria de la Ruta de Integración Latinoamericana): Conociendo el Potencial Existente como Nuevos Espacios de Desarrollo y Prácticas Innovadoras - Continuidad; y el Proyecto Gobernanzas Migratorias en Brasil: Desafíos Contemporáneos en la Agenda 2030 - Organización de las Naciones Unidas (ONU). E-mail: acdorsa@ucdb.br, Orcid: http://orcid.org/0000-0002-1120-0273

Bruno de Matos Faria: Estudiante de Doctorado en Desarrollo Local (Ciencias Ambientales) y magíster en Desarrollo Local por el Centro Universitario Augusto Motta (UNISUAM). Especialización en Docencia On-Line: Tutoría en Educación a Distancia por el UNISUAM. Grado en Arquitectura y Urbanismo por la Universidad Estácio de Sá (UNESA). Profesor ayudante en 
la UNESA, en el Curso de Arquitectura y Urbanismo e Ingeniería Civil. Coordinador y profesor auxiliar del Centro Universitario Gama e Souza (UNIGAMA), en el Curso de Arquitectura y Urbanismo e Ingeniería Ambiental y Sanitaria. E-mail: bmfarias@gmail.com, Orcid: https://orcid. org/0000-0001-9756-6999

Maria Geralda de Miranda: Postdoctorado en Políticas Públicas y Educación Humana en la Universidad Estatal de Río de Janeiro (UERJ); en Narrativas Visuales, en la Universidad Clásica de Lisboa; y en Estudios Culturales Africanos, en la Universidad Federal de Río de Janeiro (UFRJ). Doctorado en Estudios Culturales por la Universidad Federal Fluminense (UFF). Grado en Comunicación Social (Periodismo) por las Facultades Integradas Hélio Alonso (FACHA), y en Letras Clásicas y Vernáculas por la Fundación Educativa Unificada Campograndense (FEUC). Desarrolla estudios en el contexto del Desarrollo Sostenible, Educación Ambiental, Cultura y Educación. E-mail: mariamiranda@globo.com, Orcid: https://orcid.org/0000-0002-2461-7414 
